
jp0728353

Submitted April 11, 2007; Revised May 21, 2007

\title{
Scaled Density Functional Theory Correlation Functionals
}

\author{
Mohammed M. Ghouri, ${ }^{a}$ Saurabh Singh, ${ }^{a}$ and B. Ramachandran ${ }^{b^{*}}$ \\ a) Institute for Micromanufacturing, Louisiana Tech University, Ruston, LA 71272 \\ b) Chemistry, College of Engineering and Science, Louisiana Tech University, Ruston, LA 71272
}

\begin{abstract}
We show that a simple one parameter scaling of the dynamical correlation energy estimated by the Density Functional Theory (DFT) correlation functionals helps increase the overall accuracy for several local and nonlocal functionals. The approach taken here has been described as the "scaled dynamical correlation" (SDC) method [B. Ramachandran, J. Phys. Chem. A 2006, 110, 396], and its justification is the same as that of the Scaled External Correlation (SEC) method of Brown and Truhlar. We examine five local and five nonlocal (hybrid) DFT functionals, the latter group including three functionals developed specifically for kinetics by the Truhlar group. The optimum scale factors are obtained using a set of 98 data values consisting of molecules, ions, and transition states. The optimum scale factors, found using a linear regression relationship are found to differ from unity with a high degree of correlation in nearly every case, indicating that the deviation of calculated results from the experimental values are systematic, and proportional to the dynamic correlation energy. As a consequence, the SDC scaling of dynamical correlation decreases the mean errors (signed and unsigned) by significant amounts in an overwhelming majority of cases. These results indicate that there are gains to be realized from further parameterization of several popular exchange-correlation functionals.
\end{abstract}

\footnotetext{
${ }^{*}$ E-mail: ramu@latech.edu
} 


\section{Introduction}

In a recent paper $^{1}$ we explored whether it was possible to obtain accurate atomization energies for molecules by scaling the dynamical correlation energy calculated by Density Functional Theory (DFT) ${ }^{2}$ correlation functionals without significant deterioration of the structural and spectroscopic properties of the molecules. We were able to answer this question in the affirmative with reference to a small test set of neutral molecules, and two local (or pure) and two nonlocal (or hybrid) exchange functionals, all coupled to the LYP correlation functional. ${ }^{3}$ In the present work, we study the performance of a larger and more diverse set of DFT functionals, and explore the applicability of the scaling of dynamical correlation to neutral molecules, radicals, cations, anions, and transition states.

The justification for the scaling, for which we suggested the name "scaled dynamical correlation" or SDC, is very similar to that for the "scaled external correlation" or SEC method of Brown and Truhlar: ${ }^{4}$ the dynamical correlation ${ }^{5}$ has a weak geometry dependence and can be scaled by a simple constant factor in order to increase the accuracy of calculations. The difference between the SEC and SDC methods has to do with how the dynamical correlation energy is estimated. While the SEC is based on scaling the energy difference between CASSCF $^{6}$ and $\mathrm{MR}-\mathrm{CI}^{7}$ calculations, the SDC makes use of the energy difference between DFT calculations employing only the exchange $\left(E_{x}\right)$, and the full exchange-correlation $\left(E_{x c}\right)$ functionals. The justification for associating the difference between $E_{x}$ and $E_{x c}$ with dynamical correlation is provided by the work of Mok et al. ${ }^{8}$ and Gritsenko et al. ${ }^{9}$ and is further explained below.

The electron correlation energy of a molecule is defined as ${ }^{10}$

$$
E_{\text {corr }}=E-E_{\mathrm{HF}},
$$


where $E$ is the exact (non-relativistic) energy and $E_{\mathrm{HF}}$ is the Hartree-Fock limit energy. It is recognized that $E_{\text {corr }}$ is a composite quantity made up of at least two components: the "dynamical" or "external" correlation energy and the "non-dynamical" or "internal" correlation energy. ${ }^{5}$ The SEC method is based on the approximate separation between the internal and external correlation energy afforded by the combination of CASSCF and MR-CI when used with a "sufficiently large" basis set. The CAS energy is expected to include most of the nondynamical correlation while the CI calculation using the CAS solutions as references recovers a portion of the remaining (presumably mostly dynamical) correlation energy. The incomplete recovery of dynamical correlation by MR-CI -due to the twin limitations of finite one-electron basis set size and the truncation of the slow-converging CI series typically at single and double excitations (MR-CISD)- typically results in the underestimation of bond dissociation energies and overestimation of reaction barrier heights. The basis of the SEC method is to scale the difference between the CAS and MR-CISD energies by a constant factor so as to bring the total energy closer to the correct value. So, for a given nuclear geometry, the SEC-scaled energy is obtained as ${ }^{4}$

$$
E^{\mathrm{SEC}}=E^{\mathrm{CAS}}+\frac{1}{F}\left[E^{\mathrm{MR}-\mathrm{CISD}}-E^{\mathrm{CAS}}\right]
$$

where the scale factor $F$ is determined by reference to experimental bond energies.

The SDC method is based on the observation ${ }^{8,9}$ that the generalized gradient approximation (GGA) exchange-correlation functionals used in DFT also provide the means of separating dynamical and non-dynamical correlation energies but at considerably less computational cost. Gritsenko et al. ${ }^{9}$ note that "the GGA exchange functionals represent effectively not only exchange, but also the molecular non-dynamical correlation, while the $G G A$ 
correlation functionals represent dynamical correlation only (emphasis added)." Based on this idea, the SDC energy of a diatomic $\mathrm{AB}$, in analogy to Eq. (2), may be written as

$$
E^{\mathrm{SDC}}=E_{x}+f\left[E_{x c}-E_{x}\right]
$$

where $E_{x c}$ is the total energy calculated using a particular GGA exchange-correlation functional, $E_{x}$ is the energy obtained by using only the exchange part of the functional, and $f$ is the scaling factor. The difference $\Delta E_{c}=E_{x c}-E_{x}$ is taken to be a measure of the dynamical correlation energy.

The remainder of this paper is organized as follows. In Section 2, we describe our calculations and the conventions used for defining the scale factors. In Section 3, we present the results. In Section 4, we summarize the important conclusions from this work and make some observations about the limitations and consequences of the proposed scaling.

\section{Calculations}

\section{A. Optimum Scale Factors}

Before discussing the details of the calculations and examining the results, it is useful to examine some general features of the method we are about to employ. In all cases, we shall consider the energies of the molecules or ions relative to those of the neutral atoms, i.e., atomization energies (AE). The optimum SDC scale factor for a given set of atomization energies is obtained by re-arranging Eq. 3, replacing absolute energies with atomization energies, and setting $A E^{\mathrm{SDC}}=A E^{\text {expt }}$. Thus we get

$$
A E^{\text {expt }}-A E_{x}=f\left(A E_{x c}-A E_{x}\right) .
$$

It is readily demonstrated that scaling the AEs directly in this manner is equivalent to scaling the absolute energies of both the molecule and the constituent atoms using the same scale factor. 
The optimum scale factor $f$ for a set of $\mathrm{AE}$ values can now be defined in various ways, for example, as an unweighted average over the training set, a weighted average, or as the value that minimizes some statistical measure of error. We choose the last option in this work, and use linear regression (minimization of squared residuals) which is suggested by the form of Eq. 4. It is important to note that if the calculated $A E_{x c}$ values are scattered uniformly above and below the $A E^{\text {expt }}$ values, the value of $f$ will be close to unity and no advantage will result from the scaling. In the worst case scenario, the $r^{2}$ correlation of the fit will also be significantly less than unity, indicating that the linear relationship implied by Eq. 4 is not justified. On the other hand, if $A E_{x c}$ deviates systematically from $A E^{\text {expt }}$ as a function of the dynamical correlation energy, we would expect the $r^{2}$ correlation coefficient to be close to unity, and for $f$ to be less than unity (over-estimation of $\Delta E_{c}$ by the functional) or greater than unity (underestimation of $\Delta E_{c}$ ). In such cases, we can expect significant gains to result from scaling $\Delta E_{c}$. The practical implementation of such scaling in calculations would involve expressing the energy as

$$
E_{x c}^{\mathrm{SDC}}=E_{x}^{\mathrm{DFT}}+f \Delta E_{c}
$$

in the case of local (or pure) DFT functionals, and

$$
E_{x c}^{\mathrm{SDC}}=a E_{x}^{\mathrm{HF}}+(1-a) E_{x}^{\mathrm{DFT}}+f \Delta E_{c}
$$

in the case of hybrid methods that incorporate the non-local "exact" Hartree-Fock exchange.

The scale factor in our approach is applied to the entire correlation functional. A more common approach has been to scale the gradient-correction to the correlation (the so called nonlocal term), as in the venerable B3LYP ${ }^{11}$ functional

$$
E_{x c}^{\mathrm{B} 3 \mathrm{LYP}}=a E_{x}^{\mathrm{HF}}+(1-a) E_{x}^{\mathrm{Local}}+b \Delta E_{x}^{\mathrm{GGA}}+\Delta E_{c}^{\mathrm{VWN}}+c \Delta E_{c}^{\mathrm{GGA}},
$$

where optimum values of $a, b$, and $c$ were found to be $0.20,0.72$, and 0.81 by fitting to experimental data. ${ }^{12}$ Truhlar and coworkers have also investigated the effects of adjusting the 
parameter $c$ in Eq. $7 .^{13,14}$ Cafiero's scaled correlation functional for use with exact exchange $\mathrm{e}^{15}$ also belongs to this category. If, on the other hand, the SDC scaling were to be applied to LYP, an additional scale factor $f$ would multiply the entire correlation term, so that we get

$$
\Delta E_{c}^{\mathrm{SDC}}=f\left(\Delta E_{c}^{\mathrm{VWN}}+c \Delta E_{c}^{\mathrm{GGA}}\right) .
$$

This does have some consequences and we will discuss those in Section 4.

One would expect the optimum scale factors $f$ for richly parameterized hybrid methods such as B3LYP ${ }^{11}$ O3LYP,${ }^{16}$ X3LYP,${ }^{17}$ PW6B95,${ }^{18}$ PWB6K $,{ }^{18} \mathrm{M} 05,{ }^{19} \mathrm{M} 05-2 \mathrm{X},{ }^{20} \mathrm{M} 06-\mathrm{L},{ }^{21}$ and M06- $\mathrm{HF}^{22}$ to yield scale factors close to unity with high $r^{2}$ coefficients, especially if evaluated against the training set used to parameterize them. Therefore, we have not examined such functionals in the present work. On the other hand, the results presented below indicate that almost all local and most one-parameter nonlocal functionals generally benefit from SDC scaling, sometimes quite significantly. With the regression approach we have adopted, the extent to which the scaling decreases the error in calculated properties is directly related to (a) the $r^{2}$ correlation factor, and (b) the extent to which $f$ differs from unity.

\section{B. DFT functionals}

The following combinations of exchange and correlation functionals were studied: BLYP,${ }^{3,23}$ OLYP,${ }^{24}$ mPWPW91, ${ }^{25,26}$ mPW1PW91, ${ }^{25,26}$ OPW91, ${ }^{24,25}$ PBEPBE, ${ }^{27}$ PBE1PBE, ${ }^{28}$ mPW1K, ${ }^{29}$ (a version of mPW1PW91 with $42.8 \%$ exact exchange), PPWB1K $^{30}$ (a hybrid based on $44 \%$ exact exchange mixed in with mPW, and Becke's B95 correlation functional ${ }^{31}$ ), and $\mathrm{BB} 1 \mathrm{~K},{ }^{32}\left(\mathrm{~B} 88^{23}\right.$ with $42 \%$ exact exchange and the B95 correlation $\left.{ }^{31}\right)$. The last three are functionals specifically designed for kinetics by the Truhlar group by adjusting the relative amounts of exact (i.e., Hartree-Fock) exchange in the exchange part of the functional. Since the other functionals in our set do rather poorly in the location and energy of saddle points 
(sometimes completely failing to locate a saddle-point), the barrier-height calculations are done using only these three functionals.

\section{Calculations}

All calculations make use of the Pople $6-31+\mathrm{G}(d, p)$ basis set, ${ }^{33}$ which represents an excellent compromise between computational effort and accuracy in calculated geometries and electronic properties, so much so that it has been dubbed the "desert island double-zeta" (DIDZ) basis set by Truhlar. ${ }^{32}$ Calculations for a small set of molecules were also done using the $6-311++\mathrm{G}(2 d f, 3 p d)$ basis $^{34}$ to examine basis set effects on the scale factors. All calculations are performed using Gaussian $03^{35}$ using a pruned grid of 99,590 points (the "ultrafine" grid) for integral evaluations.

The computations involve (a) calculation of the "exchange only" energy, $E^{x}$, and (b) calculation of the exchange-correlation energy, $E^{x c}$. In all cases, we consider the energy of the species under consideration relative to the atoms. In the case of neutral molecules, this yields the familiar atomization energy (AE). In the case of ions, we consider the relative energy of the ion with respect to the neutral atoms and a zero-energy electron:

$$
\begin{aligned}
& \mathrm{AB}^{+}+e^{-} \rightarrow \mathrm{A}+\mathrm{B} \\
& \mathrm{AB}^{-} \rightarrow \mathrm{A}+\mathrm{B}+e^{-}
\end{aligned}
$$

The calculated results are evaluated against the experimental atomization energies for each species. Since the energy difference between $\mathrm{AB}$ and $\mathrm{AB}^{+}$is the ionization potential (IP), knowledge of the experimental AE and IP of AB is sufficient to calculate the "experimental" $\mathrm{AE}$ of $\mathrm{AB}^{+}$. Likewise, the "experimental" $\mathrm{AE}$ of $\mathrm{AB}^{-}$is obtained from the experimental $\mathrm{AE}$ and electron affinity (EA) of AB. The "experimental" AEs of transition states are calculated from the AE's of the reactants and the best-estimate barrier heights $\Delta V^{*}$ for the reactions. The 
reference values for these properties (AE, IP, EA, and $\Delta V^{\ddagger}$ ), except for the cases noted below (subsection 2.D), are taken from the extensive databases compiled by the Truhlar group. ${ }^{36}$ In order to make fair comparisons against the calculations, we have adjusted the experimental results for the experimental zero-point energies and the atomic and molecular spin-orbit (SO) splittings. $^{37}$

The basis of comparisons between methods is the mean unsigned error (MUE) and the mean signed error (MSE) for the set. In the case of neutral molecule and transition state atomization energies, we also report mean unsigned error per bond (MUEPB) to facilitate comparisons to other methods evaluated using different sets of molecules.

In every case, with three exceptions as noted below, $E_{X}$ is obtained by "turning off" the correlation part of the functional using Gaussian IOp options ${ }^{38}$ even when stand-alone exchange functionals (as in the case of B88 or OPTX) are available. The three exceptions are the mPWB95 and BB95 functionals required to implement the mPWB1K and BB1K methods, respectively. The Gaussian implementation of these methods does not permit the correlation part to be turned off as described above. So, the mPWPW91 and BLYP functionals are used, with the appropriate weights for exact exchange, to obtain $E_{x}$ in these cases. ${ }^{39}$ Some subtle differences in the LYP correlation functionals used in the BLYP and OLYP methods should also be noted. The LYP part of BLYP uses functional III of Vosko, Wilk, and Nusair, (VWN) ${ }^{40}$ for the local correlation whereas the LYP used in OLYP makes use of functional V of Ref. 40 (VWN5).

\section{Molecules, Ions, and Reactions}

The complete list of the molecules used for calculations of atomization energies are given in supporting information. The 26 ions used for ionization potential and electron affinity 
calculations are the same as those in the IP/13 and EA/13 datasets of Zhao and Truhlar. ${ }^{36,41}$ The test set for transition states and the best estimates for their barrier heights are also taken from the work of Zhao and Truhlar ${ }^{32,36}$ and consist of the 21 reactions as given in Table S4 of the Supporting Information for Ref. 32. The reference values for two barrier heights have been changed as follows. The reaction barrier for the $\mathrm{O}\left({ }^{3} \mathrm{P}\right)+\mathrm{HCl}$ of $9.8 \mathrm{kcal} / \mathrm{mol}$ used by Zhao and Truhlar is based on the S5 potential energy surface of Ramachandran et al. ${ }^{42}$ However, more recent and much more extensive computations by Ramachandran and Peterson ${ }^{43}$ leads to a benchmark barrier height of $10.6 \mathrm{kcal} / \mathrm{mol}$, and this value is used as the best estimate in the present work. The barrier for the reverse reaction is taken to be $10.45 \mathrm{kcal} / \mathrm{mol},{ }^{43}$ based on the AE for $\mathrm{OH}$ estimated to be $107.17 \mathrm{kcal} / \mathrm{mol}$ by Ruscic et al. ${ }^{44}$

The equilibrium geometries for all neutral molecules and molecular ions used in calculations of AE, IP, and EA values are the QCISD/MG3 optimized geometries provided in the Minnesota database. ${ }^{36}$ For the reaction barrier calculations, we started with the QCISD/MG3 saddle points provided in the Minnesota database, but re-optimized each saddle point with the three one-parameter exchange-correlation functionals optimized for kinetics. In these cases, the reactant and product equilibrium geometries were also found by optimization using the respective DFT functionals, starting with the QCISD/MG3 optimized geometries. 


\section{Results}

\section{A. Neutral molecules and radicals}

Four examples of SDC scaling (Eq. 4) applied to neutral molecules and radicals are shown in Figure 1. In Fig. 1(a), we plot Eq. 4 as it applies to the PBEPBE and mPWK1K functionals. The scale factors found by linear regression for these two functionals are, respectively, $f=0.906$ and 1.068 . Figure $1(\mathrm{~b})$ shows the data obtained from OLYP and OPW91 and provides an interesting comparison of the behavior of two correlation functionals coupled to the same exchange functional. The systematic way in which the calculated results deviate from the experimental results in all four cases is immediately apparent from Fig. 1. The "outliers," three of which are denoted by arrows, tend to be the same molecules in all four cases.

Table I summarizes the results of the analysis for AEs of neutral molecules and radicals for all 11 functionals. The following points are noteworthy. The scale factors for all methods

are different from unity, and the $r^{2}$ coefficients for the regression range between 0.992 and 0.996 , suggesting a high degree of correlation. With the exception of OLYP and PBE1PBE, SDC scaling results in a decrease of the MUE, ranging from $2.2 \%$ for $\mathrm{mPWB} 1 \mathrm{~K}$ to $38.9 \%$ for PBEPBE. In the two cases where scaling increases the MUE, the increases are only $1.3 \%$ for OLYP and $1.6 \%$ for PBE1PBE. Except in the case of BLYP and PBE1PBE, the scaling also decreases the absolute value of the MSE, by significant amounts in most cases. The discussion immediately below Eq. 4 is important to evaluate the significance of these observations. If the errors in the calculated AEs were randomly distributed on either side of zero, we would expect the scale factor to be very close to unity, leaving the errors essentially unchanged after scaling (see mPWB1K in Table I). We would also expect the scaled and unscaled MSE to be rather small in such a case. That this is not generally the case suggests that the errors in the energies 
calculated by these functionals are systematic, and that further gains are possible by a simple parameterization of their correlation functionals.

Another observation to be made from Fig. 1 is that in some cases (for example, mPW1K), it is possible to further reduce the errors by relaxing the constraint that the $y$-intercept of the linear fit (Eq. 4) be zero. The equation, in the case of mPW1K, then becomes

$$
\left(A E^{\text {expt }}-A E_{x}\right) / \text { a.u }=0.999464\left(A E_{x c}-A E_{x}\right)+0.015559
$$

This results in a reduction in the scaled MUE from 7.83 to $7.07 \mathrm{kcal} / \mathrm{mol}$ (an improvement of $32.9 \%$ relative to the unscaled MUE). The MSE of an unconstrained least-squares fit is, of course, zero. The relaxation of the constraint in the scaling of atomization energies has other implications which we will discuss in Section 4.

We now examine the influence of basis set size on the scale factors using a small, but representative, sample of molecules. The test set employed is the AE6, ${ }^{45}$ whose six molecules are representative of the 109 AEs in the Minnesota database ${ }^{36}$ and is also expected to be representative of the present test set. Table II summarizes the scale factors and errors over the AE6 set using the $6-31+\mathrm{G}(d, p)$ and the larger 6-311++G(2df,3pd) basis sets. The scale factors are comparable for both basis sets, indicating that the significant increase in basis set size does not compensate for the systematic deviation of calculated AEs from experimental values noted for the $6-31+\mathrm{G}(d, p)$ basis set in Fig. 1 and Table I. In other words, the decreases in the MUE reported in Table I are not all due to compensation of basis set deficiencies (although it would be impossible to avoid some compensation of basis set errors). It is also noteworthy that the scale factors for the 6-31+G(d,p) for the AE6 molecules in Table II are close to those obtained for the entire test set of neutral molecules and radicals in Table I, confirming that the AE6 set is indeed representative of the training set used in Table I. 


\section{B. Transition States, reactants, and products}

The results of applying SDC scaling to the atomization energies of transition states, reactants, and products of the 21 reactions studied (Table S4 of the Supporting Information for Ref. 32) are summarized in Table III. The data used to find the optimum scale factor for $\mathrm{mPW} 1 \mathrm{~K}$ is plotted in Figure 2, along with the linear fit which is constrained to pass through the origin. Once again, the scaling of the correlation energy results in reductions of the MUE ranging from a low of $9.9 \%$ for $\mathrm{mPWB} 1 \mathrm{~K}$ to a high of $40.1 \%$ for $\mathrm{mPW} 1 \mathrm{~K}$. The MUEs reported in this Table cannot be directly compared to those for the barrier heights or reaction thermochemistry reported for these methods because those are based on differences between calculated energies, but also because the geometries used in our case are optimized using the exchange-correlation functionals used whereas it is our impression that the Truhlar group uses QCISD/MG3 geometries for consistency when comparing different methods.

The optimum scale factors for all four methods are greater than unity, indicating that these hybrid functionals systematically underestimate the correlation energy. The high percentages of nonlocal exchange incorporated into these methods serve to overcome the welldocumented $^{29}$ tendency of most pure DFT functionals to overestimate the correlation energy of transition states, resulting in barrier heights that are too low. From Fig. 2 and Table III, it appears that these functionals may also benefit from re-parameterization of the correlation part in addition to the parameter $a$ (see Eq. 6) in the exchange part of the functional - provided the scaling does not deteriorate the energy differences between the reactants and transition states. We will examine whether this is indeed the case in Section 3.E. 


\section{Ions}

DFT methods generally have difficulties with ionization potentials (IP) and electron affinities (EA). We use the 13 ionization potentials and 13 electron affinities in the IP13 and EA13 databases ${ }^{36}$ as our test set. As described in Section 2.C, we convert all ion energies into AEs. Two examples of the behavior of the energies for SDC scaling purposes are shown in Figure 3. The summary of the results for all functionals examined is provided in Table IV. The SDC scaling improves the MUE for all except BB1K by 1.9 (PBEPBE) to $24.3 \%$ (mPWB1K). In the case of BB1K, the apparent scatter in the data (indicated by the low value of the $r^{2}$ coefficient) combined with the requirement that the fit pass through the origin results in a worsening of the MUE as a consequence of scaling. The rather dramatic increase in the numerical value of the MSE in several cases (mPW1PW91, PBE1PBE, mPW1K, and mPWB1K) can also be traced back to the systematic deviations introduced by forcing the line to pass through the origin. It is tempting to try various measures to obtain more optimum fits, such as fitting positive and negative ions separately, fitting the molecular and atomic ions separately, or relaxing the constraint that the fit should pass through the origin, so that larger reductions in the MUE and MSE can be obtained. We have verified that these expectations are indeed met if such steps are taken. For example, relaxing the constraint on the fit helps realize more dramatic improvements in most cases (but not BB1K), as shown in the last column of Table IV. Unconstrained least-squares fits, of course, yield vanishing MSE in every case. We have not reported the results of the other possible measures to reduce the errors mentioned above because such fine-tuning of scaling for a test set is not the goal of this paper, and indeed it is of dubious practical value. Our goal, rather, is to show that most pure and one-parameter hybrid DFT functionals show systematic deviations from the correct values, that such deviations are 
generally proportional to the dynamical correlation energy, and that it is possible to correct for such deviations with relatively simple measures.

\section{Overall weighted averages}

The weighted average of the MUE values (the mean MUE, or MMUE) for the 51 neutral molecules and radicals, 21 transition states (for the three kinetics functionals), and 26 ions provide a measure of the overall performance of the SDC scaling. The unscaled MMUE values range from $3.76 \mathrm{kcal} / \mathrm{mol}$ for OLYP to $8.89 \mathrm{kcal} / \mathrm{mol}$ for $\mathrm{BB} 1 \mathrm{~K}$. The scaled MMUE values range from $3.63 \mathrm{kcal} / \mathrm{mol}(\mathrm{OLYP})$ to $7.72 \mathrm{kcal} / \mathrm{mol}(\mathrm{BB} 1 \mathrm{~K})$. The percent improvement from SDC scaling ranges from $3.3 \%(\mathrm{OLYP})$ to $24.0 \%(\mathrm{mPW} 1 \mathrm{~K})$. There are no cases in which the scaling increased the MMUE. The relatively small improvement observed in the case of the OLYP functional can be attributed to the fact that the weighted average of the scale factors for OLYP is 1.001 whereas that for $\mathrm{mPW} 1 \mathrm{~K}$ is 1.112 .

\section{E. Relative energies from scaled atomization energies}

It is clear from the results presented in Tables I-IV that the deviation of calculated AEs from the experimental values is proportional to $\Delta E_{c}$ for all functionals examined, and that substantial improvements are possible by incorporating the optimum scale factor $f$ into the calculations. However, relative energies, $\Delta E$, in which errors can mutually cancel, are of more practical interest for the accurate determination of properties such as reaction barrier heights, enthalpies of reaction, IPs, and EAs. It is reasonable to assume that accuracy in AEs will automatically translate into accuracy in relative energies. However, because of statistical error inherent in determining the optimum value of $f$ applicable to a wide variety of species, the errors in the AEs of the two species whose $\Delta E$ is calculated could add rather than cancel, resulting in a magnification of the overall error. 
In Table $\mathrm{V}$, we compare the results of calculating the barrier heights $\Delta V^{*}$ and energies of reaction $\Delta E^{\mathrm{rxn}}$ from the unscaled and scaled AEs using the four functionals optimized for kinetics. The overall MUEs from the scaled AEs are close to, but actually slightly worse, than those directly calculated from the kinetics functionals. Since these three functionals are specifically parameterized to reproduce barrier heights and reaction energies accurately (rather than accurate prediction of AEs), we do not believe that this is a surprising outcome. The scaling, on the other hand, allows these functionals to deliver even greater accuracy for AEs without significant degradation of performance for barrier heights and thermochemistry.

Table VI presents a comparison of the errors IPs and EAs calculated from scaled AEs of the ions and neutral species with those directly calculated from the 10 functionals examined. The IP and EA values calculated from constrained scaling of AEs lead to lower MUEs for OLYP, OPW91, and mPWB1K while the MUE increases for most of the remaining functionals. Calculating IPs and EAs from AEs obtained from unconstrained scaling leads to reductions of MUE for a larger number of functionals, especially mPW1PW91 and PBE1PBE, and turns a rather large negative "improvement" in the case of mPW1K into a small, but, positive result.

\section{F. Scaling relative energies}

Although scaling of AEs using Eq. 4 is easier to justify on physical grounds (it is equivalent to scaling the absolute energies of molecules and constituent atoms using the same scale factor), the approach outlined above can also be applied directly to relative energies that represent properties of interest.

Let us first consider the reaction barrier heights $\Delta V^{*}$ and zero-point exclusive reaction energies $\Delta E^{\mathrm{rxn}}$. Scaling the barrier heights directly in this fashion implies that the absolute 
energies of both the transition state and the reactants are scaled using the same scale factor $f$.

Eq. (4) may be directly adapted and generalized for this case as

$$
\Delta V^{\ddagger \text { expt }}-\Delta V_{x}^{\ddagger}=f\left(\Delta V_{x c}^{\ddagger}-\Delta V_{x}^{\ddagger}\right)+c .
$$

This expression, applied to the 42 reactions in our test set (the 21 reactions as listed in Table S4 of the Supporting Information for Ref. 32 and the corresponding reverse reactions) are plotted in Figure 4 for $\mathrm{mPW} 1 \mathrm{~K}$. The reason for allowing a non-zero y-intercept $c$ should be immediately apparent from Fig. 4. The slopes and intercepts from the linear regression for the three kinetics functionals are summarized in Table VII. The reaction energies are obtained from the barrier heights as $\Delta E_{f}^{\mathrm{rxn}}=\Delta V_{f}^{\sharp}-\Delta V_{r}^{\ddagger}$, where the subscripts indicate forward and reverse reactions. We make the following observations from Table IV: (a) the $r^{2}$ correlation for the fits are much worse than the earlier instances in which the scaling was applied to atomization energies, and (b) there are substantial gains in accuracy to be attained from using Eq. (9), as indicated by the reductions in MUE. It should be noted that the unscaled MUE and MSE values for this set are somewhat larger than those reported by the Truhlar group for these methods, ${ }^{36}$ presumably because we are using saddle-point and asymptotic geometries optimized using the methods rather than the QCISD/MG3 structures provided in the Minnesota database.

At least a part of the reason for the non-zero value of $c$ is that slightly different scale factors are optimal for the transition states and the reactants. Consider the reaction

$$
\mathrm{A}+\mathrm{B} \rightarrow \mathrm{AB}^{\ddagger} \rightarrow \text { products, }
$$

where, for generality, we will assume that both A and B are molecules. Using the definition of the reaction barrier, $\Delta V^{\ddagger}=E^{\ddagger}-\left(E^{A}+E^{B}\right)$, Eq. 9 can be written in the form

$$
\Delta V^{\ddagger \text { expt }}-\Delta V_{x}^{\ddagger}=f_{1} \Delta E_{c}^{\ddagger}-f_{2}\left(\Delta E_{c}^{A}+\Delta E_{c}^{B}\right)
$$


where optimum values for the scale factors $f_{1}$ and $f_{2}$ can be found by multiple regression. We have performed such an analysis for $\mathrm{mPW} 1 \mathrm{~K}$ and obtained slightly different values $f_{1}$ for the transition states and $f_{2}$ for the reactants, which resulted in a slightly lower overall MUE than that reported in Table VII.

We next turn to the scaling of IPs and EAs directly. In each case, we are dealing with an energy difference between an ion and the neutral species. We choose to deal with both IP and EA together, as

$$
\Delta E^{\text {expt }}-\Delta E_{x}=f\left(\Delta E_{x c}-\Delta E_{x}\right)+c,
$$

as in the case of Eq. (9). However, because of our desire to treat IP and EA for both molecular and atomic ions as one set, we find $f$ by constraining the fit to pass through the origin (i.e., $c=0$ ), as shown in Fig. 5 for the cases of OLYP and BB1K. The former represents a case where the constrained linear fit represents the data reasonably well whereas such an approach is clearly suboptimal in the case of BB1K. Table VIII provides a summary of the results of scaling the IP and EA values for all functionals examined. OLYP is the best case as noted above, resulting in an $18 \%$ improvement in MUE as a result of scaling, whereas BB1K is the worst, with an increase in MUE by $17 \%$ after scaling. In the case of BB1K, it is clear from Fig. 5(b) that the MUE for the scaled IP and EA values can be reduced substantially by scaling the positive and negative ions separately. Upon doing so, but still constraining each fit to pass through the origin, two of the three kinetics functionals yield impressive decreases in the MUE for direct scaling of IP and EA: $0.48 \%$ for $\mathrm{mPW} 1 \mathrm{~K}, 19.9 \%$ for $\mathrm{mPWB} 1 \mathrm{~K}$, and $23.4 \%$ for BB1K. It is clear from the data plotted in Fig. 5(b) that further gains are possible in the case of BB1K by relaxing the constraint $c=0$ in Eq. (11) for each set. 


\section{Summary and Discussion}

We have shown that 10 popular pure and hybrid DFT functionals show systematic deviations of atomization energies from experimental values, and that these deviations are generally proportional to the dynamical correlation energy, which means that a simple one parameter scaling of correlation energy greatly increases the accuracy in many cases. That such gains are possible was demonstrated using a set of 51 neutral molecules, 26 atoms, and 42 reactions. The weighted averages of the MUEs over this test set of 98 values showed gains in accuracy ranging from 3 to $24 \%$ (Section 3.D). Such gains from scaling would be impossible to realize if the deviations of calculated energies from experimental values were random, even if such deviations are generally small for the better functionals. We also verified (see Table II) that the gains in accuracy from scaling cannot be attributed to compensating for the smaller size of the basis set used, although it is clear from the extensive benchmarking of various DFT methods performed by the Truhlar group (for example, Refs. 20 and 21) that larger (and better) basis sets

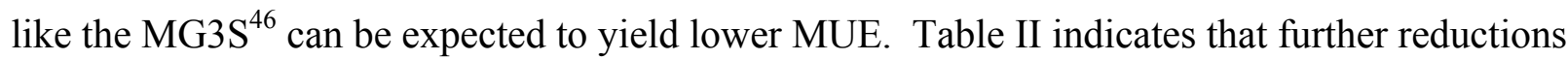
to these lower MUEs may be possible by scaling the correlation energy.

We mentioned in connection to Eq. 9 that relaxing the constraint on the least-squares fit to find the optimum scale factor $f$ (that the fit pass through the origin) had other implications. Without the constraint, Eq. 4 can be re-written as

$$
A E^{\text {expt }}=\left(A E_{x}+c\right)+f \Delta E_{c}
$$

which implies that a non-zero value of $c$ is a statistical measure of the deviations of the energy calculated by the exchange functional from the "correct" exchange value. The exchange functional in DFT contains not only the Hartree-Fock contribution to the energy but also the nondynamical correlation. ${ }^{9,24}$ In the case of atoms and atomic ions, the non-dynamical correlation is 
zero $^{24}$ and, therefore, the exchange functional should yield energies that approach the HartreeFock limit. To the best of our knowledge, only the OPTX functional of Handy and $\mathrm{Cohen}^{24}$ has been designed with this constraint (but the B88 was optimized against the HF energies for the noble gas atoms). Another well-known problem with some exchange functionals is the selfinteraction error which leads to a non-zero exchange energy for a one-electron system. Among the exchange functionals we have examined, only the B88 and OPTX are free of this error.

An item postponed from Section 2 is the consequences of scaling the local as well as the gradient-correction term in the correlation functional. A price to be paid for this is that the resulting functional will no longer tend to the correct limit as the system becomes a uniform electron gas (UEG). Conformity to the UEG limit appears to be more important for applications involving metals but whether such a constraint is necessary at all to obtain accuracy is still a matter of debate. For example, Handy and Cohen ${ }^{24}$ note that the UEG "is most unlike small atoms and molecules" and did not impose that constraint on the OPTX functional. Nevertheless, our test set does not include any metals and it is possible that an approach such as this may be limited to non-metallic systems.

The optimal scale factors for atomization energies of neutral species appear to be quite different from those required for ions. Therefore, it seems unlikely that a method with reasonable accuracy for a broad range of systems will result from the present single parameter approach. On the other hand, it should be possible, given good training sets, to use this approach to adapt existing functionals for specialized applications in which only particular types of molecules or interactions are of interest.

In the present work, we have treated the exchange and correlation energies given by the functionals as independent pieces that can be assembled to yield the final result. In practical 
implementations, the exchange contribution comes from the converged exchange-correlation density, which would be different from the density obtained by turning off the correlation part and converging the SCF iterations. While the analysis presented above is closer in spirit to the

SEC method of Brown and Truhlar, ${ }^{4}$ a slightly different approach is required to implement the scale factor in practical calculations: the $E_{x}$ used for determining the dynamical correlation energy for scaling, $\Delta E_{c}=E_{x c}-E_{x}$ must be obtained using the same density that yields the $E_{x c}$. Fortunately, the two densities appear to be fairly similar, at least for neutral species. We have applied the latter approach to the AE6 set for a few functionals and confirmed that the optimum scale factors are close to those reported in Table II for the $6-31+\mathrm{G}(d, p)$ basis set, and that comparable gains in accuracy are possible. Such scale factors can then be incorporated into the correlation functional, ${ }^{47}$ and could be the basis for the development of DFT functionals specialized for specific types of molecules or applications. We are currently evaluating the performance of a few such functionals.

\section{Acknowledgements}

We thank Professor Donald G. Truhlar and Yan Zhao for helpful discussions on the methods and training sets developed by the Truhlar group and for providing data to verify that we are implementing the kinetics functionals correctly. Use of the IBM p5-575 computers provided by the Louisiana Optical Network Initiative (LONI) is gratefully acknowledged. 


\section{References}

1 Ramachandran, B. J. Phys. Chem. A 2006, 110, 396.

2 Parr, R. G.; Yang, W. Density Functional Theory of Atoms and Molecules, Oxford University Press, New York, 1989).

Lee, C.; Yang, W.; Parr, R. G. Phys. Rev. B 1988, 37, 785.

4 Brown, F. B.; Truhlar, D. G. Chem. Phys. Lett. 1985, 117, 307.

5 Sinanoğlu, O. Adv. Chem. Phys. 1964, 6, 315.

6 Roos, B. O. Int. J. Quantum Chem. Symp. 1980, 14, 175.

7 Werner, H. -J. Adv. Chem. Phys. 1987, 69 (Part I), 1.

8 Mok, D. K. W.; Neumann, R.; Handy, N. C. J. Phys. Chem. 1996, 100, 6225.

9 Gritsenko, O. V.; Schipper, P. R. T.; Baerends, E. J. J. Chem. Phys. 1997, 107, 5007.

10 Löwdin, P.O. Adv. Chem. Phys. 1959, 2, 207.

11 Stephens, P. J.; Devlin, F. J.; Chabalowski, C. F.; Frisch, M. J.; J. Phys. Chem. 1994, 98, 11623.

12 Becke, A. D. J. Chem. Phys. 1993, 98, 5648.

13 Dahlke, E. E.; Truhlar, D. G. J. Phys. Chem. B 2005, 109, 15677.

14 Schultz, N. E.; Zhao, Y.; Truhlar, D. G. J. Phys. Chem. A 2005, 109, 11127.

15 Cafiero, M. Chem. Phys. Lett. 2006, 418, 126.

16 Handy, N. C.; Cohen, A. J. Mol. Phys. 2001, 99, 607.

17 Xu, X.; Goddard III, W. A. Proc. Nat. Acad. Sci. (US) 2004, 101, 2673; Xu, X.; Zhang, Q.; Muller, R. P.; Goddard III, W. A. J. Chem. Phys. 2005, 122, 014105.

18 Zhao, Y.; Truhlar, D. G. J. Phys. Chem. A 2005, 109, 5656.

19 Zhao, Y.; Schultz, N. E.; Truhlar, D. G. J. Chem. Phys. 2005, 123, 161103.

20 Zhao, Y.; Schultz, N. E.; Truhlar, D. G. J. Chem. Theory Comput. 2006, 2, 364.

21 Zhao, Y.; Truhlar, D. G. J. Chem. Phys. 2006, 125, 194101.

22 Zhao, Y. Truhlar, D. G. J. Phys. Chem. A 2006, 110, 13126.

23 Becke, A. D. Phys. Rev. A 1988, 38, 3098.

24 Handy, N. C.; Cohen, A. J. Mol. Phys. 2001, 99, 403.

25 Perdew, J. P. in Electronic Structure of Solids, '91; Ziesche, P.; Eschig, H., Eds. Akademie Verlag: Berlin, 1991, p. 11.

26 Adamo, C.; Barone, V.; J. Chem. Phys. 1998, 108, 664.

27 Perdew, J. P.; Burke, K.; Ernzerhof, M. Phys. Rev. Lett. 1996, 77, 3865.

28 Perdew J. P.; Ernzerhof, M.; Burke, K. J. Chem. Phys. 1996, 105, 9982. 
Lynch, B. J.; Fast, P.L.; Harris, M.; Truhlar, D. G. J. Phys. Chem. A 2000, 104, 4811.

Zhao, Y.; Truhlar, D. G. J. Phys. Chem. A 2004, 108, 6908.

Becke, A. D. J. Chem. Phys. 1996, 104, 1040.

Hehre, W. J.; Ditchfield, R.; Pople, J. A. J. Chem. Phys. 1972, 56, 2257; Hehre, W. J.;

Radom, L.; Schleyer, P. v. R.; Pople, J. A. Ab initio Molecular Orbital Theory, Wiley, New York, 1986.

Krishnan, R.; Binkley, J. S.; Seeger, R.; Pople, J. A. J. Chem. Phys. 1980, 72, 650; Frisch, M. J.; Pople, J. A.; Binkley, J. S. J. Chem. Phys. 1984, 80, 3265.

35 Gaussian 03, Revision C.02, Frisch, M. J.; Trucks, G. W.; Schlegel, H. B.; Scuseria, G. E.; Robb, M. A.; Cheeseman, J. R.; Montgomery, Jr., J. A.; Vreven, T.; Kudin, K. N.; Burant, J. C.; Millam, J. M.; Iyengar, S. S.; Tomasi, J.; Barone, V.; Mennucci, B.; Cossi, M.; Scalmani, G.; Rega, N.; Petersson, G. A.; Nakatsuji, H.; Hada, M.; Ehara, M.; Toyota, K.; Fukuda, R.; Hasegawa, J.; Ishida, M.; Nakajima, T.; Honda, Y.; Kitao, O.; Nakai, H.; Klene, M.; Li, X.; Knox, J. E.; Hratchian, H. P.; Cross, J. B.; Bakken, V.; Adamo, C.; Jaramillo, J.; Gomperts, R.; Stratmann, R. E.; Yazyev, O.; Austin, A. J.; Cammi, R.; Pomelli, C.; Ochterski, J. W.; Ayala, P. Y.; Morokuma, K.; Voth, G. A.; Salvador, P.; Dannenberg, J. J.; Zakrzewski, V. G.; Dapprich, S.; Daniels, A. D.; Strain, M. C.; Farkas, O.; Malick, D. K.; Rabuck, A. D.; Raghavachari, K.; Foresman, J. B.; Ortiz, J. V.; Cui, Q.; Baboul, A. G.; Clifford, S.; Cioslowski, J.; Stefanov, B. B.; Liu, G.; Liashenko, A.; Piskorz, P.; Komaromi, I.; Martin, R. L.; Fox, D. J.; Keith, T.; Al-Laham, M. A.; Peng, C. Y.; Nanayakkara, A.; Challacombe, M.; Gill, P. M. W.; Johnson, B.; Chen, W.; Wong, M. W.; Gonzalez, C.; and Pople, J. A.; Gaussian, Inc., Wallingford CT, 2004. http://comp.chem.umn.edu/database/

37 The adjusted, $A E^{\text {Expt }}$ is obtained as $A E^{\text {Expt }}=A E_{\text {Actual }}^{\text {Expt }}+\Delta E_{\text {zpe }}^{\text {Expt }}-\Delta E_{\text {SO,molecule }}+\Delta E_{\mathrm{SO} \text {,atom }}$, where the $\Delta E_{\mathrm{SO}}$ are degeneracy-averaged experimental multiplet energies.

38 For example, the route statement "\# OLYP/basis IOp $(3 / 78=-1)$ " results in an OPTX calculation.

39 The $E^{x}$ for mPWB1K is calculated as mPWPW91/6-31+G(d,p) IOp(3/76=0560004400) $\mathrm{IOp}(3 / 78=1)$, and that for BB1K is calculated as BLYP/6-31+G(d,p) IOp $(3 / 76=$ 0580004200) $\operatorname{IOp}(3 / 78=-1)$.

40 Vosko, S. H.; Wilk, L.; Nusair, M. Can. J. Phys. 1980, 58, 1200.

41 Zhao, Y.; Truhar, D. G. J. Phys. Chem. A 2005, 109, 5656 (supporting information; Table S3)

42 Ramachandran, B.; Schrader III, E. A.; Senekowitsch, J. S.; Wyatt, R. E. J. Chem. Phys. 1999, 111, 3862 .

43 Ramachandran, B.; Peterson, K. A. J. Chem. Phys. 2003, 119, 9590. 
44 Ruscic, B.; Wagner, A. F.; Harding, L. B.; Asher, R. L.; Feller, D.; Dixon, D. A.; Peterson, K. A.; Song, Y.; Qian, X.; Ng, C. -Y.; Liu, J.; Chen, W.; Schwenke, D. W. J. Phys. Chem. A 2002, 106, 2727.

45 Lynch, B. J.; Truhlar, D. G. J. Phys. Chem. A 2003, 107, 8996.

46 Lynch, B. J.; Zhao, Y.; Truhlar, D. G. J. Phys. Chem. A 2003, 107, 1384.

47 In Gaussian 03, this would mean using the IOp (3/78) option. 
Table I. Summary of scaling atomization energies of neutral molecules and radicals $[6-31+\mathrm{G}(d, p)$ basis].

\begin{tabular}{|c|c|c|c|c|c|c|c|c|c|}
\hline \multirow[t]{2}{*}{ Functional } & \multirow{2}{*}{$\begin{array}{l}\text { Scale } \\
\text { factor } \\
\qquad f\end{array}$} & \multirow[t]{2}{*}{$\begin{array}{c}r^{2} \\
\text { correlation }\end{array}$} & \multicolumn{2}{|c|}{$\begin{array}{c}\text { MUE } \\
(\mathrm{kcal} / \mathrm{mol})\end{array}$} & \multirow{2}{*}{$\begin{array}{c}\text { MUE } \\
\text { Improvement } \\
(\%)^{a}\end{array}$} & \multicolumn{2}{|c|}{$\begin{array}{l}\text { MUEPB }^{b} \\
(\mathrm{kcal} / \mathrm{mol})\end{array}$} & \multicolumn{2}{|c|}{$\begin{array}{c}\mathrm{MSE}^{c} \\
(\mathrm{kcal} / \mathrm{mol})\end{array}$} \\
\hline & & & Unscaled & Scaled & & Unscaled & Scaled & Unscaled & Scaled \\
\hline BLYP & 1.0324 & 0.996 & 6.67 & 5.99 & 10.2 & 1.81 & 1.62 & 0.88 & -1.90 \\
\hline OLYP & 0.9870 & 0.995 & 4.16 & 4.21 & -1.3 & 1.13 & 1.14 & -1.48 & -0.36 \\
\hline mPWPW91 & 0.9487 & 0.993 & 6.71 & 5.28 & 21.3 & 1.82 & 1.43 & -4.81 & -0.32 \\
\hline mPW1PW91 & 1.0206 & 0.996 & 5.64 & 5.13 & 9.0 & 1.53 & 1.39 & 3.94 & 2.14 \\
\hline OPW91 & 0.9469 & 0.995 & 6.37 & 5.15 & 19.3 & 1.73 & 1.39 & -3.47 & +1.20 \\
\hline PBEPBE & 0.9056 & 0.993 & 9.93 & 6.07 & 38.9 & 2.69 & 1.64 & -8.52 & -0.02 \\
\hline PBE1PBE & 0.9880 & 0.996 & 5.32 & 5.40 & -1.6 & 1.44 & 1.46 & 1.34 & 2.38 \\
\hline mPW1K & 1.0677 & 0.992 & 10.52 & 7.83 & 25.6 & 2.85 & 2.12 & 9.72 & 3.80 \\
\hline mPWB1K & 1.0045 & 0.993 & 6.22 & 6.08 & 2.2 & 1.89 & 1.87 & 3.98 & 2.69 \\
\hline BB1K & 1.0338 & 0.996 & 6.73 & 5.81 & 13.8 & 1.82 & 1.57 & 5.66 & 2.48 \\
\hline
\end{tabular}
(a) Defined as $100 \times($ Unscaled - Scaled $) /$ Unscaled.
(b) MUE/3.69
(c) $A E^{\text {Expt }}-A E^{\text {Calc }}$ 
Table II. Comparison of the scaling of the atomization energies of the AE6 molecules using two basis sets.

\begin{tabular}{|c|c|c|c|c|c|c|c|c|}
\hline \multirow[t]{3}{*}{ Functional } & \multirow{2}{*}{$\begin{array}{l}\text { Scale } \\
\text { factor } \\
f \\
\end{array}$} & \multirow{2}{*}{$\begin{array}{c}r^{2} \\
\text { correlation }\end{array}$} & \multicolumn{2}{|c|}{$\begin{array}{r}\text { MUEPB }^{a} \\
(\mathrm{kcal} / \mathrm{mol})\end{array}$} & \multirow{2}{*}{$\begin{array}{c}\text { Scale } \\
\text { factor } \\
f\end{array}$} & \multirow{2}{*}{$\begin{array}{c}r^{2} \\
\text { correlation }\end{array}$} & \multicolumn{2}{|c|}{$\begin{array}{c}\text { MUEPB }^{a} \\
(\mathrm{kcal} / \mathrm{mol})\end{array}$} \\
\hline & & & Unscaled & Scaled & & & Unscaled & Scaled \\
\hline & \multicolumn{4}{|c|}{$6-31+\mathrm{G}(d, p)$} & \multicolumn{4}{|c|}{$6-311++\mathrm{G}(2 d f, 3 p d)$} \\
\hline BLYP & 1.058 & 0.999 & 1.42 & 0.62 & 1.041 & 0.993 & 1.34 & 1.14 \\
\hline OLYP & 1.008 & 0.998 & 0.68 & 0.70 & 1.012 & 0.996 & 0.92 & 0.83 \\
\hline mPWPW91 & 0.959 & 0.998 & 1.53 & 1.03 & 0.949 & 0.986 & 1.98 & 1.51 \\
\hline mPW1PW91 & 1.029 & 0.996 & 1.64 & 1.47 & 1.018 & 0.995 & 0.99 & 0.89 \\
\hline OPW91 & 0.956 & 0.997 & 1.72 & 1.19 & 0.955 & 0.988 & 2.09 & 1.34 \\
\hline PBEPBE & 0.914 & 0.996 & 2.48 & 1.24 & 0.906 & 0.979 & 3.00 & 1.74 \\
\hline PBE1PBE & 0.995 & 0.996 & 1.47 & 1.50 & 0.985 & 0.993 & 1.19 & 0.96 \\
\hline mPW1K & 1.075 & 0.991 & 3.08 & 2.37 & 1.063 & 0.984 & 2.33 & 1.53 \\
\hline mPWB1K & 1.021 & 0.996 & 1.79 & 1.75 & 1.015 & 0.993 & 1.04 & 1.02 \\
\hline $\mathrm{BB} 1 \mathrm{~K}$ & 1.042 & 0.996 & 1.83 & 1.67 & 1.034 & 0.994 & 1.32 & 0.99 \\
\hline
\end{tabular}

(a) MUE/4.83 
Table III. Summary of SDC scaling of atomization energies of transition states, reactants and products.

\begin{tabular}{|c|c|c|c|c|c|c|c|c|c|}
\hline \multirow[t]{2}{*}{ Functional } & \multirow{2}{*}{$\begin{array}{l}\text { Scale } \\
\text { factor } \\
f\end{array}$} & \multirow[t]{2}{*}{$\begin{array}{c}r^{2} \\
\text { correlation }\end{array}$} & \multicolumn{2}{|c|}{$\begin{array}{c}\text { MUE } \\
(\mathrm{kcal} / \mathrm{mol})\end{array}$} & \multirow{2}{*}{$\begin{array}{c}\text { MUE } \\
\text { Improvement } \\
(\%)\end{array}$} & \multicolumn{2}{|c|}{$\begin{array}{l}\text { MUEPB }^{a} \\
(\mathrm{kcal} / \mathrm{mol})\end{array}$} & \multicolumn{2}{|c|}{$\begin{array}{c}\mathrm{MSE}^{b} \\
(\mathrm{kcal} / \mathrm{mol})\end{array}$} \\
\hline & & & Unscaled & Scaled & & Unscaled & Scaled & Unscaled & Scaled \\
\hline $\mathrm{mPW} 1 \mathrm{~K}$ & 1.0570 & 0.996 & 5.78 & 3.46 & 40.1 & 1.27 & 0.76 & 6.23 & 1.29 \\
\hline mPWB1K & 1.0136 & 0.998 & 2.47 & 2.22 & 9.9 & 0.54 & 0.49 & 1.95 & 0.71 \\
\hline $\mathrm{BB} 1 \mathrm{~K}$ & 1.0330 & 0.998 & 3.37 & 2.11 & 37.3 & 0.74 & 0.46 & 3.56 & 0.56 \\
\hline
\end{tabular}
(a) MUE/4.56
(b) $A E^{\text {Expt }}-A E^{\text {Calc }}$ 
Table IV. Summary of SDC scaling applied to the atomization energies of 26 ions from the IP13 and EA13 databases.

\begin{tabular}{|c|c|c|c|c|c|c|c|c|}
\hline \multirow[t]{2}{*}{ Functional } & \multirow{2}{*}{$\begin{array}{l}\text { Scale } \\
\text { factor } \\
\quad f\end{array}$} & \multirow[t]{2}{*}{$\begin{array}{c}r^{2} \\
\text { correlation }\end{array}$} & \multicolumn{2}{|c|}{$\begin{array}{c}\text { MUE } \\
(\mathrm{kcal} / \mathrm{mol})\end{array}$} & \multirow{2}{*}{$\begin{array}{c}\text { MUE } \\
\text { Improvement } \\
(\%)^{a}\end{array}$} & \multicolumn{2}{|c|}{$\begin{array}{c}\mathrm{MSE}^{b} \\
(\mathrm{kcal} / \mathrm{mol})\end{array}$} & \multirow{2}{*}{$\begin{array}{c}\text { MUE } \\
\text { Improvement } \\
(\%) \\
\text { (Unconstrained) }\end{array}$} \\
\hline & & & Unscaled & Scaled & & Unscaled & Scaled & \\
\hline BLYP & 0.9387 & 0.970 & 4.01 & 3.88 & 3.1 & -0.66 & 0.43 & 5.3 \\
\hline OLYP & 1.0268 & 0.989 & 2.89 & 2.65 & 8.7 & 1.46 & 0.98 & 21.9 \\
\hline mPWPW91 & 0.9002 & 0.969 & 3.91 & 3.68 & 6.0 & -3.08 & -1.14 & 12.4 \\
\hline mPW1PW91 & 1.0786 & 0.968 & 4.55 & 4.23 & 7.0 & -2.03 & -3.59 & 43.0 \\
\hline OPW91 & 1.0209 & 0.983 & 3.36 & 3.13 & 6.9 & -0.32 & -0.73 & 10.3 \\
\hline PBEPBE & 0.8864 & 0.963 & 3.84 & 3.77 & 1.9 & -2.51 & -0.36 & 2.7 \\
\hline PBE1PBE & 1.0753 & 0.977 & 3.94 & 3.59 & 8.7 & -1.48 & -2.93 & 43.6 \\
\hline $\mathrm{mPW} 1 \mathrm{~K}$ & 1.1936 & 0.938 & 7.28 & 6.77 & 7.0 & -1.42 & -5.30 & 35.7 \\
\hline mPWB1K & 1.1645 & 0.979 & 5.00 & 3.79 & 24.3 & 0.67 & -2.29 & 25.4 \\
\hline $\mathrm{BB} 1 \mathrm{~K}$ & 0.7978 & 0.848 & 9.47 & 11.17 & -17.9 & -4.99 & -0.14 & -18.2 \\
\hline
\end{tabular}

(a) Defined as $100 \times($ Unscaled - Scaled $) /$ Unscaled.

(b) $A E^{\text {Expt }}-A E^{\text {Calc }}$ 
Table V. Errors in reaction barriers $\Delta V^{\star}$ and energies of reaction $\Delta E^{\mathrm{rxn}}$ calculated using unscaled and scaled atomization energies.

\begin{tabular}{lcccc}
\hline \multirow{2}{*}{ Functional } & \multicolumn{2}{c}{ MUE } & \multicolumn{2}{c}{ MSE } \\
& Unscaled & Scaled & Unscaled & Scaled \\
\hline mPW1K & 1.49 & 1.60 & 0.47 & 0.74 \\
mPWB1K & 1.67 & 1.69 & 0.68 & 0.75 \\
BB1K & 1.65 & 1.67 & 0.49 & 0.66 \\
\hline \hline
\end{tabular}


Table VI. Statistical errors in ionization potentials and electron affinities calculated and using atomization energies scaled by constrained and unconstrained fits (Table IV).

\begin{tabular}{|c|c|c|c|c|c|c|}
\hline \multirow[t]{2}{*}{ Functional } & \multicolumn{2}{|c|}{$\begin{array}{c}\text { MUE } \\
(\mathrm{kcal} / \mathrm{mol})\end{array}$} & \multirow{2}{*}{$\begin{array}{c}\text { MUE } \\
\text { Improvement } \\
(\%)^{a} \\
\text { (Constrained) }\end{array}$} & \multirow{2}{*}{$\begin{array}{c}\mathrm{MSE}^{b} \\
\text { Unscaled }\end{array}$} & \multirow{2}{*}{$\begin{array}{c}(\mathrm{kcal} / \mathrm{mol}) \\
\text { Scaled }\end{array}$} & \multirow{2}{*}{$\begin{array}{c}\text { MUE } \\
\text { Improvement } \\
(\%)^{a} \\
\text { (Unconstrained) }\end{array}$} \\
\hline & Unscaled & $\begin{array}{c}\text { Scaled } \\
\text { (Constrained) }\end{array}$ & & & & \\
\hline BLYP & 3.73 & 4.43 & -18.5 & 0.45 & -0.99 & -19.2 \\
\hline OLYP & 3.20 & 2.73 & 14.5 & -0.65 & -0.04 & 19.4 \\
\hline mPWPW91 & 3.45 & 3.43 & 0.7 & -0.48 & -1.07 & 10.5 \\
\hline mPW1PW91 & 3.39 & 3.87 & -14.2 & -9.80 & -1.14 & 8.4 \\
\hline OPW91 & 3.61 & 2.83 & 21.7 & -1.57 & -0.42 & 26.2 \\
\hline PBEPBE & 3.04 & 3.12 & -2.5 & -0.24 & -0.34 & 0.8 \\
\hline PBE1PBE & 3.17 & 3.29 & -4.0 & -1.78 & -0.51 & 5.5 \\
\hline $\mathrm{mPW} 1 \mathrm{~K}$ & 4.26 & 6.13 & -43.6 & -2.89 & -3.18 & 1.5 \\
\hline mPWB1K & 3.53 & 3.25 & 8.0 & -2.12 & 0.46 & -4.1 \\
\hline $\mathrm{BB} 1 \mathrm{~K}$ & 8.32 & 9.73 & -16.9 & 3.92 & -1.38 & -17.5 \\
\hline
\end{tabular}

(a) Defined as $100 \times$ (Unscaled - Scaled)/Unscaled.

(b) $A E^{\text {Expt }}-A E^{\text {Calc }}$ 
Table VII. Summary of SDC scaling of reaction barrier heights. The linear fit is not constrained to pass through the origin.

\begin{tabular}{|c|c|c|c|c|c|c|c|c|}
\hline \multirow[t]{2}{*}{ Functional } & \multirow{2}{*}{$\begin{array}{l}\text { Slope } \\
f\end{array}$} & \multirow{2}{*}{$\begin{array}{l}\text { Constant } \\
\text { (a.u) } \\
c \times 10^{3}\end{array}$} & \multirow[t]{2}{*}{$\begin{array}{c}r^{2} \\
\text { correlation }\end{array}$} & \multicolumn{2}{|c|}{$\begin{array}{c}\text { MUE } \\
(\mathrm{kcal} / \mathrm{mol})\end{array}$} & \multirow{2}{*}{$\begin{array}{c}\text { MUE } \\
\text { Improvement } \\
(\%)\end{array}$} & \multicolumn{2}{|c|}{$\begin{array}{c}\mathrm{MSE}^{a} \\
(\mathrm{kcal} / \mathrm{mol})\end{array}$} \\
\hline & & & & Unscaled & Scaled & & Unscaled & Scaled \\
\hline \multicolumn{9}{|c|}{ A. Reaction barriers and reaction energetics } \\
\hline mPW1K & 1.5292 & 7.533 & 0.884 & 1.49 & 1.03 & 30.6 & +0.45 & -0.09 \\
\hline mPWB1K & 1.5171 & 6.321 & 0.827 & 1.68 & 1.32 & 21.5 & +0.64 & -0.01 \\
\hline $\mathrm{BB} 1 \mathrm{~K}$ & 1.4035 & 6.118 & 0.834 & 1.66 & 1.27 & 23.5 & +0.46 & +0.01 \\
\hline \multicolumn{9}{|c|}{ B. Barrier heights $\Delta V^{\ddagger}$ (forward and reverse) only } \\
\hline mPW1K & & & & 1.43 & 0.97 & 32.3 & 0.99 & 0.00 \\
\hline mPWB1K & & & & 1.53 & 1.18 & 22.6 & 1.16 & 0.09 \\
\hline BB1K & & & & 1.44 & 1.18 & 18.2 & 0.87 & 0.00 \\
\hline \multicolumn{9}{|c|}{ C. Reaction energetics $\Delta E^{\mathrm{rxn}}$ (forward) only } \\
\hline $\mathrm{mPW} 1 \mathrm{~K}$ & & & & 1.61 & 1.17 & 27.7 & -0.64 & -0.28 \\
\hline mPWB1K & & & & 1.99 & 1.59 & 19.8 & -0.39 & -0.22 \\
\hline BB1K & & & & 2.10 & 1.46 & 30.7 & -0.34 & 0.04 \\
\hline
\end{tabular}

(a) $A E^{\mathrm{Expt}}-A E^{\mathrm{Calc}}$ 
Table VIII. Summary of SDC scaling directly applied to IP and EA. The linear fit is constrained to pass through the origin.

\begin{tabular}{lccccccc}
\hline \hline Functional & $\begin{array}{c}\text { Scale } \\
\text { factor }\end{array}$ & $\begin{array}{c}r^{2} \\
\text { correlation }\end{array}$ & \multicolumn{2}{c}{$\begin{array}{c}\text { MUE } \\
(\mathrm{kcal} / \mathrm{mol})\end{array}$} & $\begin{array}{c}\text { MUE } \\
\text { Improvement } \\
(\%)\end{array}$ & $\begin{array}{c}\text { MSE }^{a} \\
\text { (kcal/mol) }\end{array}$ \\
\hline BLYP & 0.9343 & 0.950 & 3.73 & 3.86 & -3.3 & 0.45 & 0.50 \\
OLYP & 1.089 & 0.984 & 3.20 & 2.62 & 18.1 & -0.65 & -0.71 \\
mPWPW91 & 0.859 & 0.972 & 3.45 & 3.04 & 12.0 & -0.48 & -0.22 \\
mPW1PW91 & $0 . .938$ & 0.972 & 3.39 & 3.40 & -0.3 & -1.93 & -1.80 \\
OPW91 & 1.017 & 0.975 & 3.61 & 3.51 & 2.6 & -1.57 & -1.59 \\
PBEPBE & 0.884 & 0.974 & 3.04 & 2.91 & 4.4 & -0.24 & -0.03 \\
PBE1PBE & 0.963 & 0.974 & 3.17 & 3.28 & -3.5 & -1.78 & -1.69 \\
mPW1K & 0.986 & 0.958 & 3.90 & 3.92 & -0.7 & -2.89 & -2.83 \\
mPWB1K & 0.864 & 0.850 & 3.53 & 3.15 & 10.9 & -2.12 & -2.17 \\
BB1K & 0.812 & 0.769 & 8.32 & 9.70 & -16.5 & -3.92 & -1.73 \\
\hline \hline
\end{tabular}

(a) Defined as $100 \times($ Unscaled - Scaled $) /$ Unscaled. 

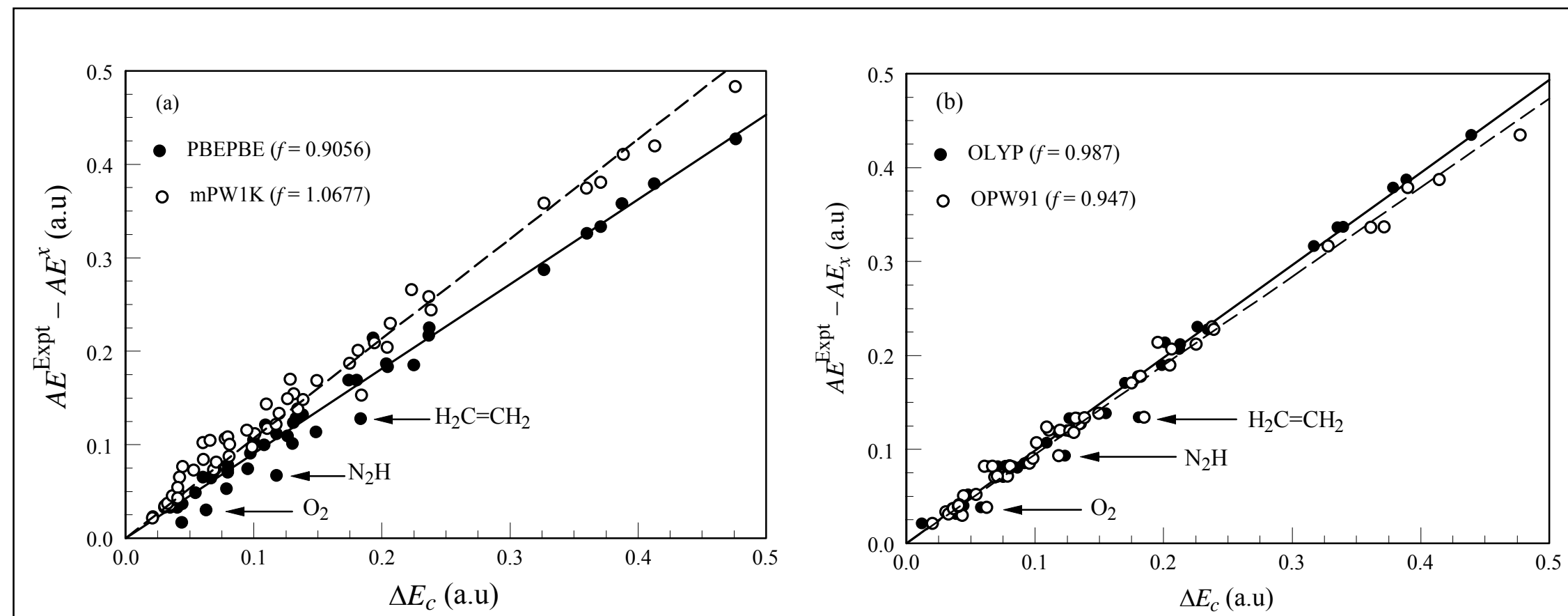

Fig. 1. SDC scaling applied to neutral molecules and radicals. (a) PBEPBE and mPW1K, and (b) OLYP and OPW91. The SDC scale factors and the linear fits are also shown. The diagonal of each figure (not drawn) represents $f=1.000$. 


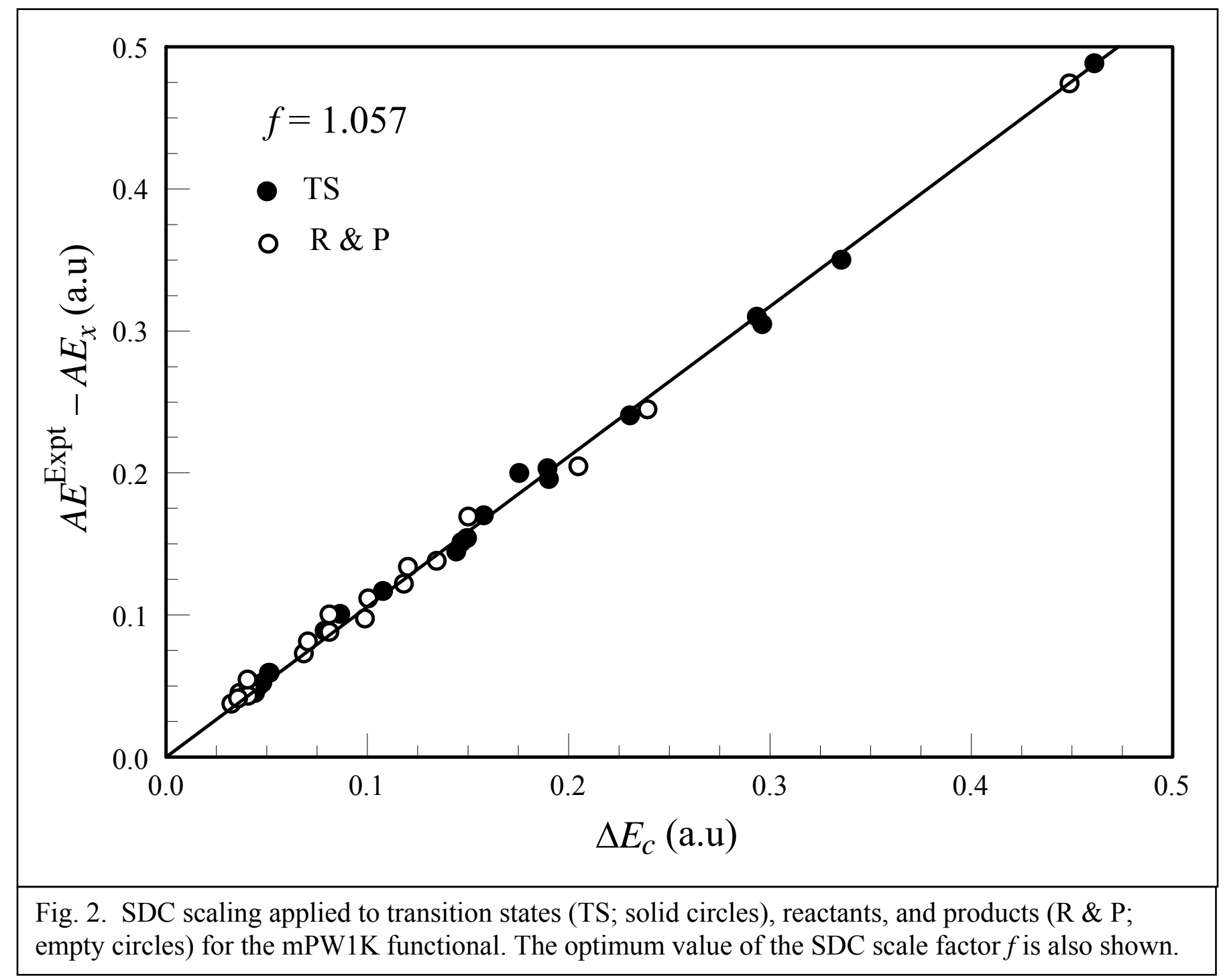



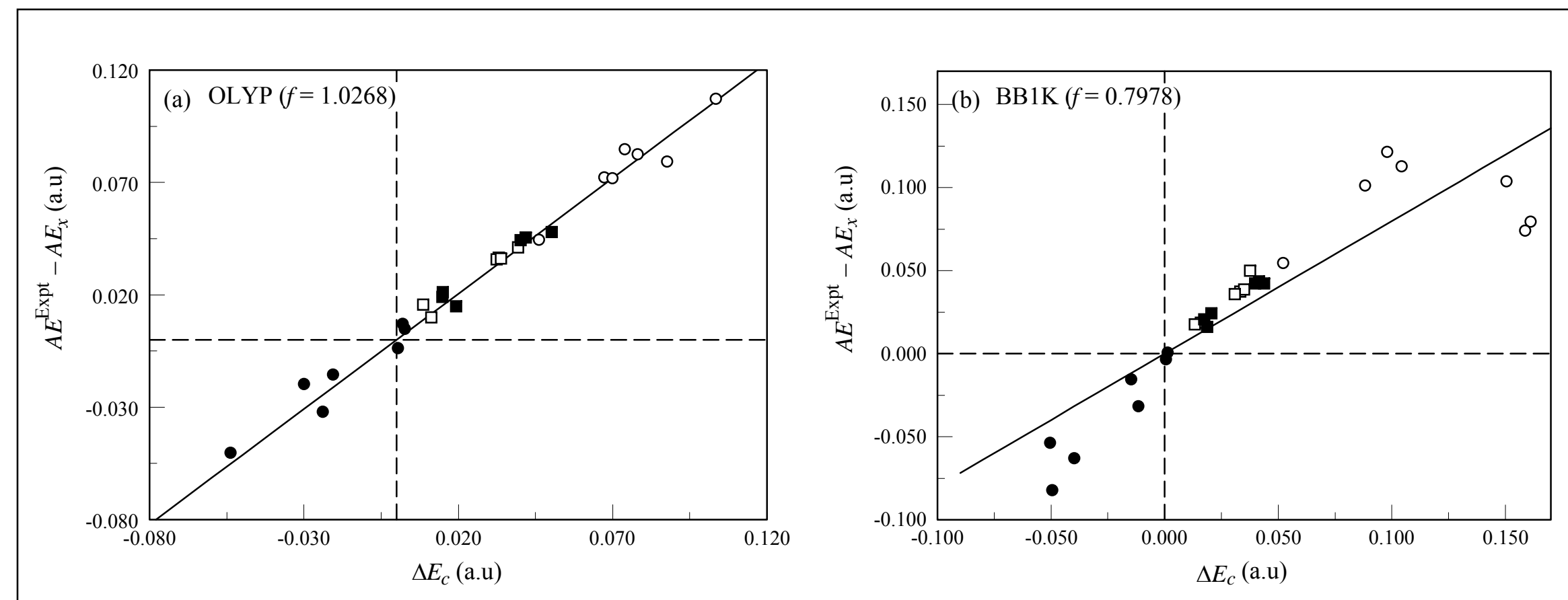

Fig. 3. SDC scaling applied to the atomization energies of 26 ions from the IP13 and EA13 database for (a) OLYP and (b) BB1K functionals. Solid circles and squares represent molecular and atomic cations, respectively, while empty circles and squares represent molecular and atomic anions. 


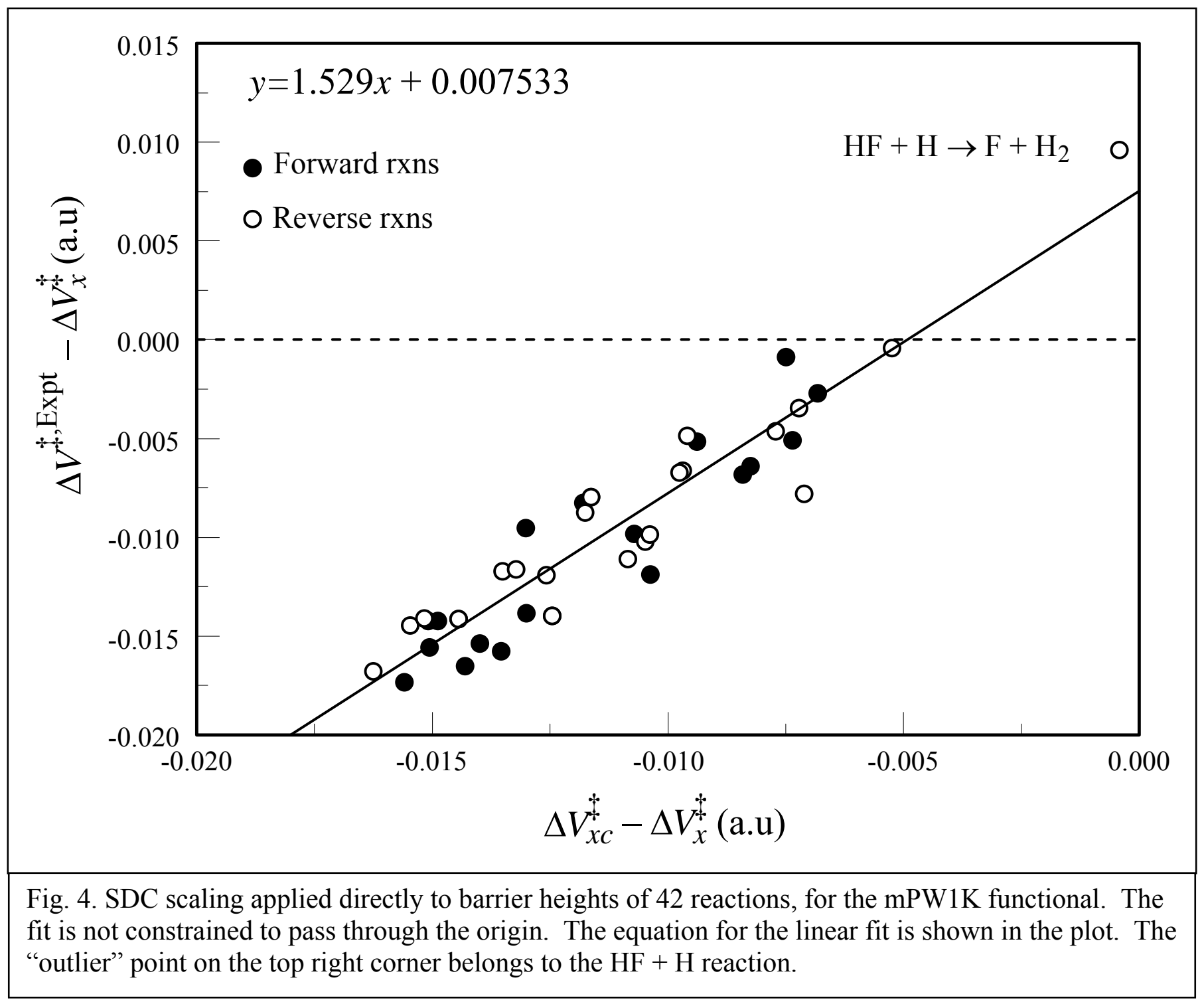




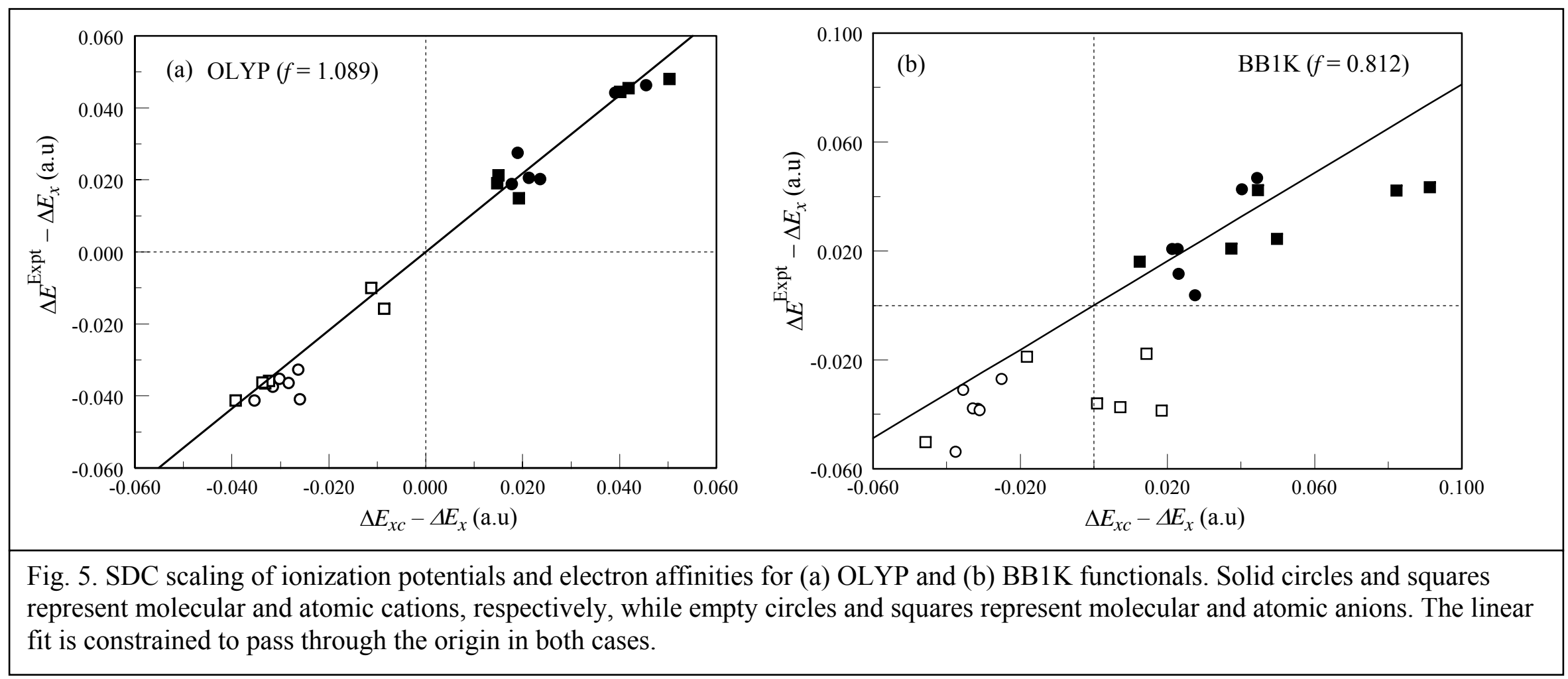

